\title{
13th Technical Conference on Hurricanes and \\ Tropical Meteorology, AMS, 1-5 December 1980, Miami Beach, Fla.
}

\author{
Robert C. Sheets ${ }^{1}$ \\ Program Chairman
}

\begin{abstract}
The 13th Technical Conference on Hurricanes and Tropical Meteorology was held in Miami Beach, Fla., during 1-5 December 1980. The special emphasis of this conference was placed upon hurricanes in recognition of the 25th anniversary of the formation of the National Hurricane Research Project. Participants came from around the world and 167 people registered for the conference. The first six sessions of the conference were devoted to invited survey or overview papers focused upon advancements in hurricane research and prediction during the past 25 years.
\end{abstract}

\section{Special sessions}

Session 1 dealt with the history of hurricane research. Robert H. Simpson (founder of the National Hurricane Research Project (NHRP)) pointed out that planning for a major hurricane research program to examine questions raised by flights through hurricanes in the late 1940 s and early 1950 s had been under way for several years before it was approved. Unfortunately, it took the disasters created by Hurricanes Carol, Edna, and Hazel in New England and the Middle Atlantic States in 1954, affecting in one year the constituencies of more senators and congressmen than ever before, to provide the motivation for funding such a research program. R. Cecil Gentry (former Director of the National Hurricane Research Laboratory (NHRL)) recalled how the right man (Simpson) happened to be in the right place (a Congressional subcommittee hearing) at the right time (after some of the disasters of 1954). After Simpson's scheduled testimony was completed, he was asked if he had anything more to say and he was "forced to present a hurricane research plan that he just happened to have with him at the time." The result was that a research program centered around airborne data collection systems was created. Through yeoman efforts and the cooperation of several key individuals within the Department of Commerce and the Department of Defense, a group of 21 employees, four research aircraft with meteorological probes, and the first airborne digital systems for recording meteorological data during penetrations of hurricanes were assembled in less than 12 months' time.

The initial research flight into a hurricane occurred on 13 July 1956. Essentially all meteorological sensor and data sys-

\footnotetext{
${ }^{1}$ National Hurricane Center, 1320 S. Dixie Highway, Coral Gables, Fla. 33146.

0003-0007/81/050633-09\$06.25

( 1981 American Meteorological Society
}

tems failed on this flight. Things were looking up on the second flight day, when most systems worked reasonably well. However, the recorded data were contained on more than 2000 punch cards, which were scattered over the wet runway when the technician removing them from the plane stumbled on the ladder. Noel LaSeur, Research Flight Director on this flight, pointed out that the cards were not numbered sequentially, and furthermore, noise problems entered random numbers in other elements, resulting in some of the cards never being properly sorted. From this auspicious beginning, LaSeur pointed out, we have come to the point where airborne capabilities now exist through NOAA's Research Facilities Center "to acquire data on many scales, ranging from cloud microphysical structure, boundary layer turbulent fluxes, convective-scale organization and intensity, and synoptic-scale structure and interactions." Illustrations of these advancements were given by Joanne Simpson, Herbert Riehl, Jose Colón, Charles Jordan, and Peter Black.

Joanne Simpson discussed the evolution of the investigation of hurricane cloud systems. She stated that the very important early NHRP documentation of time and space variations of clouds in Hurricane Daisy (1958) was obtained "from airborne radar and laborious photogrammetry from time-lapse cameras," while for Hurricane Frederic (1979), "a series of cloud-top contour maps has been constructed by stereo mapping from products of two GOES satellites on which are superimposed isopleths of radar reflectivity" from the research aircraft and digitized land-based radar. These research aircraft not only provided quantitative, detailed horizontal and vertical radar reflectivity characteristics of Hurricane Frederic's clouds, but also provided detailed microphysical data along with thermal and kinematic quantities. Simpson stated that these types of data sets, coupled with models, "and other tools, can be used to calculate the storm's requirements on high $\theta_{E}$ air, and variations in penetrative tower activity can be related to circulation changes."

Herbert Riehl discussed surface interaction as related to hurricane growth and maintenance. He stated that some of the early theories on convective overturning of the tropical atmosphere as a means of formation and maintenance of hurricanes were found wanting based upon aircraft and other observations. He cited incidents of ". . . as much as 1 meter of rain per day . . . without any unusual or even with less than normal wind speed at the surface." He then pointed out that observations of boundary layer show that "air flowing into a hurricane center maintains constant temperature in spite of rapidly decreasing pressure." The question then arises as to how this isothermal expansion can be maintained, and the time-honored answer has been large heat transfers from the ocean to the atmosphere. The question has 
then arisen whether heat for this isothermal expansion could be supplied by other processes. Two possible sources are the "downward transfer of latent heat released in the upper part of the storm" and the "dissipation of kinetic energy in the bottom layer leading to conversion to heat." Riehl stated that his calculations from aircraft observations have shown these contributions to be small. He stated that much more research was needed in this area and that the present capabilities on NOAA's research aircraft offered opportunities for collecting definitive data in this area of interest.

Jose Colón discussed the evolution of the description of the wind structure of the hurricane vortex. Early records describe these "monstrous storms, their winds, rainfall, and mountainous seas," and people's experiences during the passage of well-developed tropical cyclones. These early visual observations were followed by records from land-based instrumentation, early penetrations of storms by aircraft in the late 1940 s and early 1950 s, and finally by the highly instrumented research aircraft that evolved from the National Hurricane Research Project.

Jordan and Gray acknowledged that great advancements had been made in the documentation and description of the inner core of hurricanes, but stressed areas where much more definitive information is needed to understand the processes taking place. Of particular interest to Jordan are the

vertical circulation patterns in the upper troposphere, the tropopause structure over and near the eye, and the tilt of the eye wall with height. In addition . . . the variation of the core structure as a function of stage of development of the storm and . . . the role of concentric wall clouds which are often observed in intense storms.

(Later discussions during the conference indicated that some excellent digitized vertical and horizontal radar and other data sets were collected in Hurricanes David (1979), Frederic (1979), and Allen (1980) during occurrences of concentric eye walls and rapid changes in intensity. These data sets are being analyzed at NHRL.)

Gray stated that "there has been substantial advancement in our understanding of hurricane structure and genesis over the past 25 years." However, he indicated that there is a

need to distinguish between the relevant physical processes of the inner and outer regions of the hurricane . . . The hurricane research flights have primarily advanced our knowledge of the hurricane's inner structure. Hurricane genesis, intensity change, and motion (the major forecast parameters), however, appear to be primarily related to surrounding environmental parameters. Data coverage in this region has not been adequate and should be increased. Despite the large knowledge gained during the last 25 years, we still have a ways to go to develop satisfactory physical theories for hurricane genesis and intensity change.

New observational tools, such as air-droppable bathythermographs from research aircraft and moored ocean buoys, have been used to document the spatial and temporal distribution of the ocean response to the passage of hurricanes. Peter Black and Russell Elsberry discussed these effects, which have been revealed by observations and models, including the generation of cold wakes that persist for several days after the passage of a storm and can be modulated by storm-induced internal waves. These observational studies and mixed layer models have indicated the existence of complex interactions between the advective and mixing processes in the region where maximum temperature decreases tend to occur. If generated regions of cooler surface water are widespread enough, they may significantly lower latent and sensible heat fluxes from the ocean to the air, possibly affecting the given storm or a later storm that may cross the path of the earlier storm.

Tetsuya Fujita discussed some of his observational work with landfall hurricanes. He showed results of analyses from Hurricane Frederic, in which many swathlike areas of extreme damage occurred, while adjacent areas experienced much less damage. These damage patterns were observed not only near the coastline, but also well inland. Many local residents attributed the extreme damage to tornadic activity. However, Fujita's analyses indicated only a few tornado occurrences and attributed most of the severe damage to "downburst" windlike phenomena associated with severe eye wall and rainband convection.

Vincent Oliver summarized the evolution of the meteorological satellite as a tool for hurricane forecasting and research. He stated that the first meteorological satellite (TIROS-I) was put into orbit on 1 April 1960 and obtained its first hurricane picture soon after launch. All succeeding satellites obtained views of tropical storms in their various stages. Satellite technology advanced over the years, with various techniques being developed to determine storm position, intensity, and rainfall, as well as research advancements that are ". . . showing how tropical cyclone development is related to changes in the westerlies." Tropical cyclones should never again go undetected.

Overviews of various aspects of the evolution of the theory and modeling of the tropical cyclone were given by $\mathrm{K}$. V. Ooyama, H. L. Kuo, and Richard Anthes. Ooyama states that ". . . the general structure and energetics of the system and basic components of the supportive mechanisms were fairly well documented by the instrumented aircraft observations and diagnostic interpretation of the data by the early 1960s." The prognostic theory, however, developed much more slowly. He stated that when a viable theory finally emerged, modelers proceeded to parameterization of cumulus convection in order to reduce the multi-scale interaction problem to one that could be handled mathematically. $\mathrm{He}$ further stated that

Although mathematical simplicity of certain parameterization schemes was enticing . . . more confusion than enlightenment ensued as the mathematics overran the illdefined physics. Even with the numerical simulation of a mature tropical cyclone, the intuitive parameterization of whole clouds became an albatross on the modeler's neck that was inevitably to be discarded once the goal of modeling shifted from conceptual understanding . . . to quantitative simulation ... .

Ooyama illustrated this evolutionary process through the eyes of someone on another planet trying to build a special steam engine and the tracks to run it on. 
Kuo further tracked the modeling problem from . . . the introduction of the CISK concept and the development and application of deep cumulus parameterization in axisymmetric hurricane modeling in the late 1950 s and early 1960 s . . . to more recent direct calculations of condensation without cumulus parameterization in the symmetric model and the 3-dimensional approaches in the late 1970s.

Anthes's discussions were centered around the 3-dimensional modeling of tropical cyclones, with emphasis upon operational models for hurricane motion prediction. The fact that these operational models need to run in a time frame sufficient to provide usable forecast guidance means that they cannot contain some of the sophistication of the research models, such as high resolution. (Research models often run in more than real time- $18 \mathrm{~h}$ to make a $12 \mathrm{~h}$ forecast.) Furthermore, as these operational models progress, the time comes when they start to utilize real data and major initialization problems arise. However, progress has been made to the point that tests from the National Meteorological Center's (NMC) medium fine mesh (MFM) hurricane model have shown that under certain situations, real data inputs can cause significant improvements in the results. Also, recent operational runs in regions where data coverage is good have shown significant improvements in longer-range forecasts (more than $48 \mathrm{~h}$ ). These results are quite encouraging.

Sanders, Ramage, and Neumann discussed forecast motion predictions over the past 25 years. They pointed out that steering concepts have been and continue to be the primary basis for most forecasting techniques. Sanders indicated that tropical cyclone forecasts were probably better than their mid-latitude counterparts. However, he stated that even greater accuracy was required "because of the smaller size and extreme devastation of the region of damaging weather." Ramage stated that "forecast accuracy trends for Miami, Guam, and Hong Kong have been similar for the past 20 years. Modest improvements [in forecast accuracies] were shown until the early 1970 s, followed by modest deterioration."

Neumann developed a forecast difficulty index (FDI), based upon such factors as degree of persistence and storm latitude, longitude, speed, and intensity, to take into account variations in forecast difficulty for use in assessing year-toyear trends. The application of this index to the past 25 years of data in the Atlantic, Gulf of Mexico, and Caribbean indicated a modest improvement during the first 15 years and nearly no change during the past decade. That is, the deterioration shown in the general statistics in recent years apparently is due to a higher percentage of more difficult storms to forecast during this period as compared to the earlier period, rather than a decrease in forecasting skill levels. Neumann attributed the small improvements in forecast skill levels to improved positioning through improved reconnaissance, satellite technology, and objective guidance. Most of the statistical and dynamical models have similar average errors. However, Sanders pointed out that "the real value of these statistical and especially the dynamical approaches resides in their ability to deal with the relatively few anomalous tracks." It is here that more evaluation is needed.
Neil Frank acknowledged the improvements in understanding and prediction of hurricane motion during the past 25 years, but stressed that this

rate of improvement . . . is not keeping pace with escalating demands for more accurate predictions. Evacuation of highly vulnerable areas [the historic means of avoiding large loss of life during the occurrence of landfall hurricanes] is no longer feasible in some areas, due to increased population concentrations coupled with our present forecast limitations.

The question then arises as to whether or not the hurricane warning service could be made more effective without significant improvements in hurricane intensity and motion forecasts. Frank cited studies by behavioral scientists that showed that even with the short lead times that are presently provided, many people, including those in authority, delay their actions beyond what could be critical periods. Delays of as much as $6 \mathrm{~h}$ between the time of the warnings for Hurricanes David and Frederic (1979) and the time when appropriate actions began were observed. He cited the need for more of these types of studies so that warning procedures could be adjusted to optimize the desired response from affected communities.

The presentation that probably received the most attention during the special sessions was that given by the Associate Administrator of NOAA, George Benton. Benton stated that despite austere budgeting during the past few years, especially for research, he had found that "well thought out, carefully planned, and coordinated programs which had clear, concise goals with significant benefits" were generally received favorably in the budget process. $\mathrm{He}$ further stated that programs that did not meet all of these criteria, which may have been approved in less austere periods, were doomed to failure today. He pointed out that no major new comprehensive initiative relative to hurricane research had been proposed for several years and felt that the time was now right to put together such an initiative. Considerable interest in his proposal was expressed by members of research laboratories, operational units, and the academic community involved in tropical cyclone research and prediction.

\section{Regular sessions ${ }^{2}$}

The regular sessions consisted of more than 100 papers, with the great majority concerned with hurricanes. Essentially every scale of motion was addressed in both theoretical and modeling as well as observational studies. In addition, sessions were held on the Florida Area Cumulus Experiment (FACE), GARP Atlantic Tropical Experiment (GATE), and Monsoon Experiment (MONEX). These results will be summarized first, followed by those of the hurricane studies.

The experimental seeding phase of FACE was completed in 1980. Several stratifications of types of days continue to be investigated for use in evaluation of preliminary results of

\footnotetext{
${ }^{2}$ Extracted from summaries provided by session chairpersons.
} 
rainfall statistics for seeded versus nonseeded days and for determining factors detrimental or favorable to rainfall enhancement. A paper by Lopez et al. presented radar time and area composites and synoptic data, which together show the importance of the south Florida sea-breeze interaction with synoptic-scale disturbances and the regional circulation. Six recurring patterns emerged that depend mostly on the position of the Atlantic Ridge relative to the Florida peninsula. Days with explosive cloud growth had a stable layer present at low levels and late afternoon sea-breeze development.

Gannon examined variations of soil moisture as a possible factor influencing rainfall. Preferred regions of genesis were over or adjacent to the boundary regions between "wet" and "dry" centers. McCumber and Pielke used a primitive equation (PE) model with a layered soil approach to evaluate soil parameterization in a mesoscale model. They found that in the Florida sea-breeze simulation, the differences in latent heat flux were greatest in midday and were not so large for sensible heat flux. Soil type appeared to alter the southwest extent of sea-breeze convergence along the west coast of Florida.

Watson and Blanchard found that on "wet" days the surface convergence peaked at $1330 \mathrm{EST}$ and the rainfall peaked somewhat later. In some cases, the maximum rainfall rates occurred at the time of maximum positive divergence. The weaker the low-level wind speed, the stronger the convergence and the heavier the rainfall. In addition, Holle and Watson found that on the average, visible clouds appeared $25 \mathrm{~min}$ after the first convergence and rapid cloud growth occurred $60 \mathrm{~min}$ after initial convergence.

The GATE session papers summarized several studies concerning the convective structure of various systems moving off of Africa. Some of the findings were:

1) Convective cores are often highly tilted in the vertical and have low-level reflectivity maximums (Gamache et al.).

2) Large stratiform precipitation areas with prominent radar bright bands developed over the trailing region of early summer squall lines and accounted for much of the precipitation (Rappaport and Houze).

3) Variations of dry static energy are in the same sense from one line to another, but major differences in moist static energy exist (Szoke et al.).

4) Long narrow lines of precipitation convection formed along the leading edge of large dust plumes that emerged from the desert regions of Africa in the ridge region of traveling synoptic-scale waves (Marks).

In addition, an objective analysis of winds and derived kinematic fields during GATE Phase III has been completed which "contains fascinating rich details of evolving and propagating disturbances . . . never seen in previous analyses . . . of the same data" (Ooyama).

Results from the monsoon studies included:

1) Thermal patterns and wind fields with baroclinic structure were most pronounced on the equatorward side of cirrus cloud shields and were associated with monsoon depressions, while gradients decreased near the tropical easterly jet (Astling et al.).

2) The 3-dimensional structure of a monsoon depression showed a concentrated warm region above the surface center in the lowest layer becoming more extensive in the middle troposphere (Douglas).

3) Strong regular diurnal cycles of precipitation were observed with a maximum offshore at 0600 LST and onshore at $2200 \mathrm{LST}$. These areas of stratiform rain falling from anvil clouds strongly resemble GATE cloud cluster precipitation (Houze et al.).

4) Most of the precipitation above the $0^{\circ} \mathrm{C}$ level in convective and stratiform monsoon precipitation systems during both winter and summer MONEX was in the form of ice (supercooled liquid water was scarce) (Herzegh et al.).

Several sensitivity and/or diagnostic modeling studies investigating processes favorable or unfavorable for tropical cyclone development were reported. Many of these studies were centered around cumulus effects, momentum transports, and boundary layer processes. An example of these studies was the use of a 3-dimensional mesoscale boundary layer model to study the influence of a surface inhomogeneity on the heat, moisture, and wind fields. In this test, the influence of a heated island generated strong turbulence that resulted in the formation of cumulus-type clouds. Similar tests without the heated island gave no well-organized convective features (Beniston).

A simple mixed layer model applied to a composite of a number of GATE wave disturbances showed the important control of convective downdrafts on the large-scale average depth of the mixed layer in regions surrounding precipitation systems. These results could have significant effects upon the construction of large-scale models (Johnson). Results that are perhaps complementary were obtained by use of a 3-dimensional cloud model initiated with composite soundings obtained in the inner and outer regions of developing Atlantic tropical cyclones. The results showed the importance of the rearrangement of horizontal momentum by the simulated cumulonimbus for the deepening of a tropical cyclone (Cotton and Tripoli). Cumulus momentum mixing was shown again to be an important mechanism that demonstrated its capability for inducing a cooperative interaction between a cumulus cloud population and the hurricane-scale motion (Mak).

Numerical simulations of tropical cyclone development with an axisymmetric PE model showed the relative significance of cumulus momentum rearrangement and cumulus heating as growth mechanisms during tropical cyclone genesis (Fingerhut). Another PE model used with an idealized wind field from GATE successfully simulated a tropical cyclone genesis. Emphasis was placed upon the role of horizontal and vertical shear in the development or nondevelopment process (Tuleya and Kurihara). Cumulus effects on the rotational wind were found to be efficient in modifying the quasibalanced flow of tropical disturbances - the more asymmetric they are, the more efficient they are. Cumulus effects appearing in the thermodynamic equation are inefficient in 
modifying the quasi-balanced flow-the more asymmetric they are, the less efficient they are (Schubert).

The production of vertical vorticity by the turning of the existing background vorticity associated with planetary boundary layer flow and the earth's rotation was discussed by reference to conceptual and laboratory models (Morton). Results of a laboratory experiment on the deflection of vertical jets in cross-flows has provided insight into the vorticity dynamics of interacting flows. Momentum transport by interacting atmospheric flows was also addressed in a model of cloud ensembles. A six-hour simulation with GATE data indicates that cloud momentum transfer by updrafts and downdrafts tends to lower the African easterly jet by about $100 \mathrm{mb}$.

Several advances have been made in the cumulus convection-environment thermodynamic interaction problem with

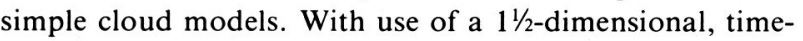
dependent, axisymmetric cloud model, a population of clouds is considered embedded within a mesoscale region and it is found that there is an important dependence of computed mass fluxes on the cloud environment moisture stratification (Huffman). Another model has been developed (Wong) that uses the observed log-normal distribution of tropical clouds and includes the effects of cloud life cycles and mesoscale downdrafts to predict successfully GATEobserved rainfall rates and liquid water, heat source, and moisture sink profiles. A semiprognostic application of the Arakawa-Schubert parameterization theory modified to include the effects of convective-scale and mesoscale downdrafts (Payne) shows that certain deficiencies in some predictions using the no-downdraft form of the model, notably excessive drying in the lower troposphere, are substantially remedied by the incorporation of downdrafts. The application of cumulus parameterization schemes to a class of largescale models, such as those used in climate studies, could benefit from the application of a simplified, quadratic-functional representation of the vertical thermodynamic profiles along with the use of simple cloud mass flux profiles (Albrecht).

A modified version of Sundquist's symmetric model equations for hurricane development was integrated with and without Reynold's stresses using parameterized eddy fluxes of momentum derived from McBride and Gray's composite wind data for three stages of hurricane development. With Reynold's stresses equal to zero, the model failed to develop an organized vortex when the initial conditions corresponded to a cloud cluster or depression. With observed Reynold's stress values, the model rapidly developed a mature hurricane in every case (Pfeffer and Chella).

The cyclostrophic and hydrostatic adjustment processes were investigated for local removal and/or redistribution of mass and angular momentum in simple one- and multilayered vortex flows. Results indicate that mass transfer taking place inside or outside of the radius of maximum tangential wind has little effect upon the wind speeds. These results indicate that other factors need to be considered for possible modification of the tropical cyclone vortex, directed at reducing the maximum wind speeds by alteration of mass flow (Smith).

A nonhydrostatic model initiated from a motionless, conditionally unstable atmosphere has been developed as an ex- tension of investigations that have explored the sympathetic relationship between cumulus and hurricane-scale motions. Whereas former experiments have relied on hydrostatic constraints, the new model is completely elastic, allowing temporally damped sound waves. The results of this investigation are too preliminary to compare with hydrostatic simulations, but are encouraging in the respect that a tropical vortex was generated from a quiet initial state (Willoughby).

Further findings from model results indicate that improved forecasts can be expected from PE models that include real data. However, initialization problems need to be overcome. Sensitivity tests to study the impact of massmomentum imbalances on hurricane track forecasts were conducted by altering the divergence fields. The result was relatively small differences in mean vortex motion over two days for one model. Nevertheless, small oscillations of path remained on the reruns and were identified as internal modes remaining in the integrations (Fiorino).

Chang used real data in an axisymmetric model to attempt to improve intensity forecasts. He found that the inclusion of surface winds derived from Seasat provided no significant improvements. However, using surface winds as well as the upper-level winds substantially improved the forecasts. It is also encouraging that relatively accurate hurricane track forecasts appear to be attainable with dynamic models of moderate computer requirements. A two-way interaction nested grid model developed by Harrison was shown to produce results of comparable or better accuracy than operational forecasts. The margin of improvement became most striking at $48 \mathrm{~h}$ and beyond. Furthermore, comparisons of three models, one a rather simple coarse mesh system run by the Navy and the others fine mesh systems run by the Navy and National Weather Service, showed a tendency to support the theoretical concept that complex, high resolution models can provide more accurate forecasts. However, even the coarse mesh model showed surprisingly credible performance. At the same time, there appears little doubt that forecasts are improved in data-rich areas where synoptic-scale features are well defined. A study by Neumann and Pelissier showed that $24 \mathrm{~h}$ forecast errors of tropical cyclone motion over the Caribbean and the Gulf of Mexico are about onehalf as large as those for the North Atlantic. Other comments made during these presentations pointed out that the NMC MFM model forecast errors for tropical cyclone motion were highly dependent upon surrounding data fields and that, like the NEPRF model, the most striking forecast improvements came at $48 \mathrm{~h}$ and beyond.

Several assessments of operational hurricane track forecasting were made for operational statistical and dynamical models, as well as for official forecasts. Analyses showed that in the Atlantic, tropical cyclone forecast errors are described by a multimodal, bivariate normal distribution and not by a unimodal distribution. That is, there are at least two basic types of storms, those that move on a relatively steady track with no rapid or erratic changes in direction or speed (persistence and analog techniques work well) and those that go through less constrained motions. Unqualified use of unimodal distribution assumptions significantly underestimates error density in the $50 \%$ and below range and overestimates error density in the 50-95\% range. This factor should be taken into account in the probability forecasts presently in 
use or contemplated (Crutcher, Neumann, and Pelissier). It was also shown that even for difficult forecast cases, such as recurvature, the hurricane forecaster generally has the ability to improve on the skill of most models (Leftwich).

Several comments were made that perhaps we are reaching or have reached a plateau concerning forecast improvements, particularly from statistical models and for shortterm forecasts. These observations were supported partially by a study by Neumann and Pelissier that shows that little, if any, improvement in the ability to predict $24 \mathrm{~h}$ tropical cyclone motion has been observed over the past decade. Any improvements in these time periods will most likely come from improved definitions of past and present storm motion and the ability to incorporate this information into the various models. Refinements of models, such as the Navy's analog model TYAN, have been made by developing a series of regression equations that adjust the analog forecasts based largely on past storm motion (Peterson and Elsberry). A similar scheme has been developed for the Navy's HATRACK geostrophic steering model (Gilchrist and Elsberry).

Another method using a quasi-Lagrangian computational scheme with higher order functions has been developed by Acoltzi and Garcia. This new scheme is being applied to Gulf of Mexico and eastern Pacific Ocean cases.

The potential for disasters associated with tropical cyclones continues to escalate as population densities increase in vulnerable coastal areas. In addition, large economic losses are incurred because of uncertainties in forecast storm tracks and intensities, making action decisions difficult for responsible officials and the public in general. An intensive research program has been initiated to try to improve forecasts and warnings, with initial efforts directed at short-term forecasts using high quality detailed observations of wind, pressure, and thermal fields provided to the forecaster and operational models in real time via a reconnaissance aircraftsatellite-computer linkup. Initial evaluations indicate that forecasts and warnings can be improved by these detailed and timely data fields and that results from Hurricanes David (1979) and Frederic (1979) support this premise (Sheets).

Navy programs also have been directed at improving action decisions by providing the decision-maker with quantitative information on the likelihood of specified conditions associated with given tropical cyclone events. These programs include probabilities of selected wind categories out to $72 \mathrm{~h}$ in advance of a forecast event for various geographic locations, given a particular storm, present strength, motion, and forecast (Jarrell and Brand; Brand et al.). In addition, selected ports in the western North Atlantic and Gulf of Mexico are being evaluated as to their suitability for hurricane havens (Turpin and Brand).

Several observational studies were presented concerning tropical cyclone structure, motion, and intensity. A study by Chan showed that averages of $200 \mathrm{mb}$ and $900 \mathrm{mb}$ winds were correlated with $24 \mathrm{~h}$ changes in motion. Integrated tropospheric temperature gradients also were found to be useful predictors of speed changes. Chan proposed a track forecast scheme based upon these parameters. The required data may be obtained from satellite-derived information, and since more conventional mid-level data, generally relied upon for steering current determinations, are becoming less available or reliable, this technique could prove quite valuable in the future if it stands up to the test of time. Hunter also proposed a forecast technique based upon satellite data; this time, cloud top temperatures. Tests on independent data for selected cases gave mixed but encouraging results for longerterm forecasts. Dvorak used satellite analyses as a means of documenting what other studies have suggested using different means; that is, changes in baroclinicity in the westerlies may be used in a prognostic sense for strengthening or weakening disturbances in the subtropics, depending upon the relative positions of the mid-latitude and tropical cloud systems. Brody et al. and Rodgers et al. used equivalent blackbody temperature data obtained from satellites to estimate storm intensity. These studies were basically refinements of a system developed by Gentry, but now included time factors obtained from geostationary satellites rather than orbiting satellites. Time smoothing of cloud top temperatures seemed necessary using these data, and deep penetrative convection is perhaps a good indicator of future intensity changes. These investigations noted that inner core convection is higher for intensifying storms than for weakening storms and that a $33 \mathrm{~h}$ lag between the time of maximum active convection and maximum storm intensity appeared to exist. This delay in intensification seems to be consistent with studies by Black and Wirfel of extreme convective elements in hurricanes, sometimes referred to as "super cells." They suggest that such events occur in nearly $20 \%$ of all tropical cyclones, tend to last $12-36 \mathrm{~h}$, remain basically in the same relative position to the storm center, perhaps inhibit intensification of the vortex during their existence, and generally are followed by rapid vortex intensification after cessation of the intense, generally highly asymmetric, convective activity.

Núñez (Max Eaton Award winner) used composite data to study intensity changes in hurricanes. His results, at least in a qualitative sense, seem to support the observations cited previously; that is, he found that supergradient, or at least less subgradient, winds were observed for intensifying disturbances when compared to steady state or filling systems. He hypothesized that the intensity change occurred as a result of the adjustment response of the mass to the wind field.

Synoptic-scale influences on hurricane structure and intensity were also reported. Falls studied interactions between Hurricanes David and Frederic and concluded that outflow from David weakened Frederic while east of the Antilles as the storms came too close together, and later interactions with cold lows and asymmetries of an upper-level anticyclone over Frederic were associated with an eddy import of negative angular momentum and areas of relatively large generation of kinetic energy, which he hypothesized acted as a trigger for Frederic's rapid reintensification. Brotak showed several cases of northeastern United States tropical cyclones interacting with mid-latitude baroclinic zones and Vincent showed that apparently deep convective clouds act as a source of synoptic-scale kinetic energy in interacting tropical-extratropical cyclones. Wind speed errors of as much as $10 \mathrm{~m} / \mathrm{s}$ in $12 \mathrm{~h}$ could be expected in prediction models that do not represent the distribution and intensity of heat release associated with these deep convective systems. Thompson and Lynch showed that even a weak subtropical cyclone located between a tropical storm and a mid-latitude trough created heavy rain and flooding due to interaction. One sta- 
tion recorded 27 in of rain. Merrill used Gulf ellipse mass computations to try to infer intensity changes of a developing system. Correlations with intensity were relatively good initially, but poor later, as an intense mesoscale system affected the ellipse computations. Bosart showed how the passage of a mesoscale rainstorm across the United States and into the western Atlantic in July 1977 led to the development of a minimal tropical cyclone, which two days later became a major extratropical cyclone. It was noted that tropical cyclone genesis from land-developed meso-convective complexes also has been observed in other global locations.

As is generally the case, analysis techniques were debated often during the conference. The greatest disagreements, or at least vocal expressions, of the pros and cons of various techniques usually occurred between strong proponents of compositing techniques and what can be inferred from such studies, and proponents of case studies using limited data and how representative these results are. Questions have always arisen about the statistical characteristics of composite data sets. Gray and Mielke et al. presented the first statistical tests of the Colorado State University tropical cyclone data set composites. Random subsets of data were composited and compared to test significances of observed features. The results generally were quite favorable and offered promise of improved interpretations of differences between composites of groups of storms and various stratifications. A general consensus seemed to be that composite studies are very useful in pointing the direction for investigation, but that case studies were needed for specific types of systems when sufficient data existed to study the detailed energetics.

The first composite analysis of Southern Hemisphere tropical cyclones showed surprisingly large quantities and excellent distributions of data in the Australian region. It appears that this data set will be quite useful for improving our knowledge of larger-scale aspects of these storms (Holland).

Operational forecast and warning processes, refined numerical prediction models for storm motion and intensity, and storm surge models now require or will soon require accurate surface or near-surface wind fields in tropical cyclones. Many techniques for measurement of these winds or for analysis and calibration of developed techniques for deriving these wind fields have generated considerable interest. Near-surface wind fields over the ocean in the high wind speed area of the tropical cyclone are difficult to obtain directly and usually are obtained by extrapolation of aircraft-measured winds calibrated and combined with sparse ship and buoy observations or computed from low-level cloud motions. More recent techniques use surface roughness and resultant reflectivities to infer these winds. Hawkins et al. reported on Seasat-derived winds in tropical cyclones that appeared quite good ( $\mathrm{rms}$ errors of about $2 \mathrm{~m} / \mathrm{s}$ ) after correcting for attenuation. However, the structure of the inner core of the tropical cyclone was not well resolved due to poor spatial resolution. Cardone and Greenwood used a planetary boundary layer model to derive a surface wind field. They found that use of Seasat winds, the minimum sea level pressure in the storm, and the radius of maximum winds resulted in excellent definition of the surface wind field, agreeing well with surface analyses derived from aircraft and surface data. Ross and McLeish used Cardone's derived winds as input into a directional wave prediction model.
Comparisons were made with satellite radar images and good agreement was found in spectra as well as directional turning, suggesting that swell as well as locally generated wind waves were imaged. Jones et al. compared airborne microwave scatterometer and radiometer measurements with direct wind observations to test the feasibility of remote sensing of surface winds. Results were quite good up to wind speeds of $45-50 \mathrm{~m} / \mathrm{s}$, although some problems occurred in heavy rain areas. Powell used surface observations and winds extrapolated from aircraft measurements to derive surface wind fields and changes for Hurricane Frederic as it approached and made landfall near Mobile, Ala. He found large discontinuities at the coast, the rotation of the strongest inflow from the right rear to the right front quadrant, and the change from outflow to weak inflow in the left rear quadrant as the storm approached and made landfall. Rinehart et al. used digitally recorded time-lapse radar data at landfall to derive detailed motion vectors for Hurricane Frederic. Variations at the coastline were quite in evidence. Session attendees were quite impressed by the detail obtained from this conventional radar data. Studies by Mehta and others revealed swaths of damage as Frederic made landfall, but also revealed that great variability in damage occurred, depending upon the type of construction. Such information can be used to reconstruct wind fields.

A study by Purvis of hurricane-spawned tornadoes also suggested significant discontinuities at landfall. He found that $70 \%$ of these tornadoes occurring in the South Carolina area occurred on the north coastal plain and hypothesized that sand dunes, unique to that area, enhance boundary layer convergence and thereby play a significant role in tornado production in this area. He pointed out that such tornadoes are generated for weak and strong storms alike and even for offshore storms skirting the coast.

Thomasell showed results from an objective analysis scheme that utilizes winds from various sources, including aircraft, satellite, surface, etc., and derives a wind field that is internally consistent. Interpolated winds are required to conform to the vorticity of a modeled hurricane with a specified intensity. Results compared quite well with observed fields.

Other observations of storm structure as revealed primarily by satellite products included stereo views of Hurricane Frederic obtained from simultaneous observations from GOES East and GOES West, revealing details of the convective structure of the inner core (Hasler and Rodgers). Also, thermal structure data obtained from TIROS-N and NOAA-6 sounders were found to compare well with conventional data. RMS differences of less than $2 \mathrm{~K}$ were observed for most of the troposphere for clear soundings, with larger errors for cloudy regions (Gruber). Soundings in Hurricane David were comparable with radiosonde data, except in the low levels where inadequate vertical resolution limited microwave retrievals (Grody and Shen). Refinements of these systems should improve significantly analyses over the data-sparse tropical regions.

Kidder used NIMBUS- 6 and TIROS-N thermal sounding data to examine radial temperature gradients in selected tropical cyclones. He used this thermal data to calculate vertical vorticity differences. He proposed that these measurements could be used for prediction of tropical cyclone intensity changes. 


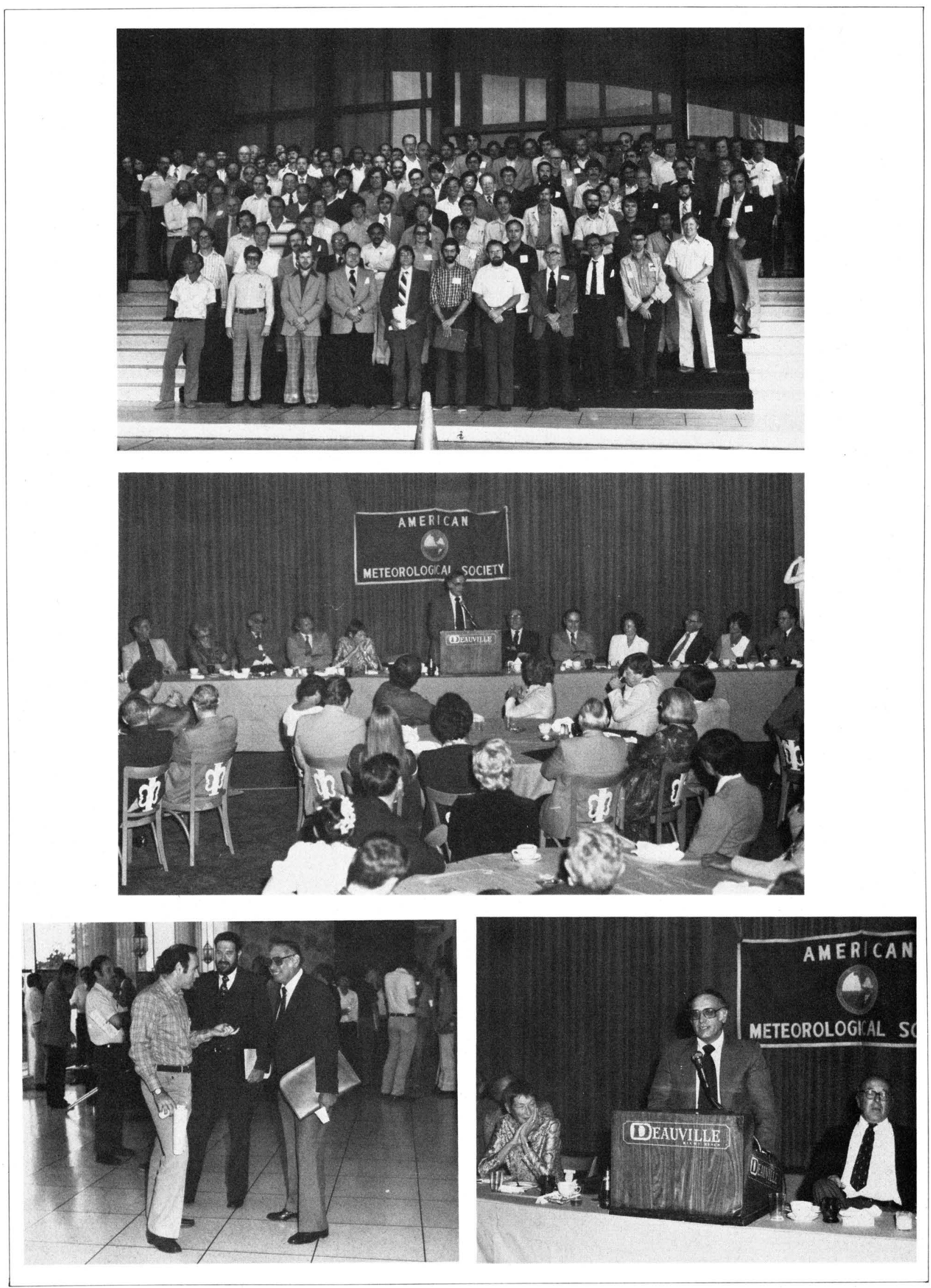


Comparisons of hurricane convective elements with GATE and other convection was made by Zipser et al. They found that despite great differences between hurricane convective element and GATE convective element environments, their convective elements were quite similar in terms of radar reflectivities, water content, vertical motion, and thermodynamic structure. Similarly, $Z-R$ relationships derived for several samples in Hurricanes Anita (1977), David (1979), and Frederic (1979) were remarkably similar when stratified by altitude, convective region, and storm intensity, with some characteristics quite similar to findings in GATE (Willis and Jorgensen). Another interesting paper on the convective structure of hurricanes was presented by Hawkins et al. They found that careful examination of many timelapse radar depictions of hurricanes revealed that eye walls often contained straight line segments rather than the generally accepted curved structure. Lewis first observed these characteristics, which often aligned themselves in a polygon arrangement in the eye wall. Hawkins then noticed similar straight line segments with abrupt angular changes in rainbands instead of the smooth spiral bands usually described. Several examples were shown from both radar and satellite products. In addition, a time-lapse movie presentation of a hurricane showed what appeared to be waves breaking through the eye wall in the radar depiction. This paper generated considerable discussion of these findings.

One of the more difficult and unanswered problems in tropical meteorology is the process whereby tropical cyclones are formed or generated from waves. Many waves are formed and travel across tropical waters each year, but only a few form tropical cyclones. Many studies have been made to investigate wave processes and three such studies were reported on at this conference. In one case, a scale analysis was applied to the vorticity equation including the effect of cumulus convection. The results indicated that the large-scale vertical velocity is nearly balanced by the cumulus mass flux, so that the vertical motion in the environment is small. Observations confirmed this analysis as representative of easterly waves (Cho et al.). The selective response of the tropical atmosphere to meridionally-propagating planetary waves also was studied using a nondivergent model. The model used a constant zonal wind plus a stationary Rossby wave, with sinusoidally-varying meridional wind velocity. The most interesting cases are those for which one of the excited modes has a frequency nearly equal to that of a Rossby wave with respect to the basic flow. In that case a strong resonant response may result (Wilson). A model also was presented that diagnoses the linear wave structure in response to a specified heat source with a given frequency and zonal wave number. The mean zonal wind and cumulus mass flux in both latitudinal and vertical dimensions were used and the primitive equations were used directly, rather than an equivalent mid-latitude beta plane. These generalizations are expected to lead to more realistic wave structure than for previous models, as well as facilitate comparison with both observations and various parameterizations of cumulus momentum transports (Stevens).

Studies of large-scale influences appear encouraging for determining longer-range predictions of tropical cyclone activity. For instance, results of a study of the interannual variability of hurricane incidence during the last 80 years show that there are recognizable two and one-half- and five-year cycles of hurricane incidence and tracks that are associated statistically with the strength of the Atlantic Subtropical High and its quasi-biennial oscillation (Shapiro). Also, active and inactive periods of tropical cyclone frequency in one hemisphere can be rather well related to simultaneous middle-latitude and tropical general circulation patterns in both hemispheres (Love). For specific situations, extensive statistical tests indicate that the tropical cyclone development potential of western Pacific disturbances can be well related to the analyses of low-level vorticity and convergence (Lowe). Also, large-scale flow changes that result from tropical cyclone passage in the western Atlantic and the western Pacific indicate that the wake area of a tropical cyclone tends to be more favorable for cyclogenesis than the same region before storm passage (Frank).

Response to hurricane warnings continues to be of great concern to officials responsible for warning and action. Much misinformation is often circulated before, during, and after a hurricane threat. NOAA has an active preparedness program to inform the general public about hurricane hazards. A study by Sanders of the Myrtle Beach, S.C., area after Hurricane David passed nearby, however, reveals that much still remains to be done in this effort.

Thirty-five percent of the people interviewed indicated that they had had prior hurricane experience. Even with the public information disseminated, only $75 \%$ of the surveyed people seemed cognizant of the wind threat associated with hurricanes and, remarkably, only $35 \%$ were aware of the storm surge hazard. Considerable discussion followed this paper and flowed into other areas. It was noted that conflicting information is often distributed by the media. Interpolations, extrapolations, and sometimes independent assessments and forecasts are given at variance with official information. Such discrepancies seem to be on the increase as media competition increases. It is feared that such conditions will result in improper, delayed, or no actions taking place when hurricanes threaten unless some solution is found to this problem.

(Top) Group picture of conference attendees. (Middle) Banquet speaker, Richard Hallgren, Director of NWS. Seated at head table, left to right, are Neil Frank, Director of NHC; Mrs. Laura Gentry; Stanley Rosenthal, director of NHRL; Hugo Bezdek, Director of AOML; Joanne Simpson, Chief, Tropospheric Branch, NASA/Goddard Space Flight Center; Richard Hallgren; Fred Sanders, Massachusetts Institute of Technology and Master of Ceremonies; Arnold Gruber, MSL; Mrs. Mickey Rosenthal; Robert H. Simpson, former Director of NHC and NHRP; Mrs. Velma Frank; and R. Cecil Gentry, former Director of NHRL. (Bottom left) (Left to right) Arnold Gruber, MSL; Alan Weinstein, Chairman of the Selection Committee; and Stanley Rosenthal, Director of NHRL. (Bottom right) (Left to right) Joanne Simpson, Chief, Tropospheric Branch, NASA/Goddard Space Flight Center; Richard Hallgren, Director of NWS; and Fred Sanders, Massachusetts Institute of Technology. 


\section{A.I.R. INC. INTRODUCES A NEW STANDARD OF QUALITY AND PERFORMANCE IN ATMOSPHERIC SOUNDING SYSTEMS}

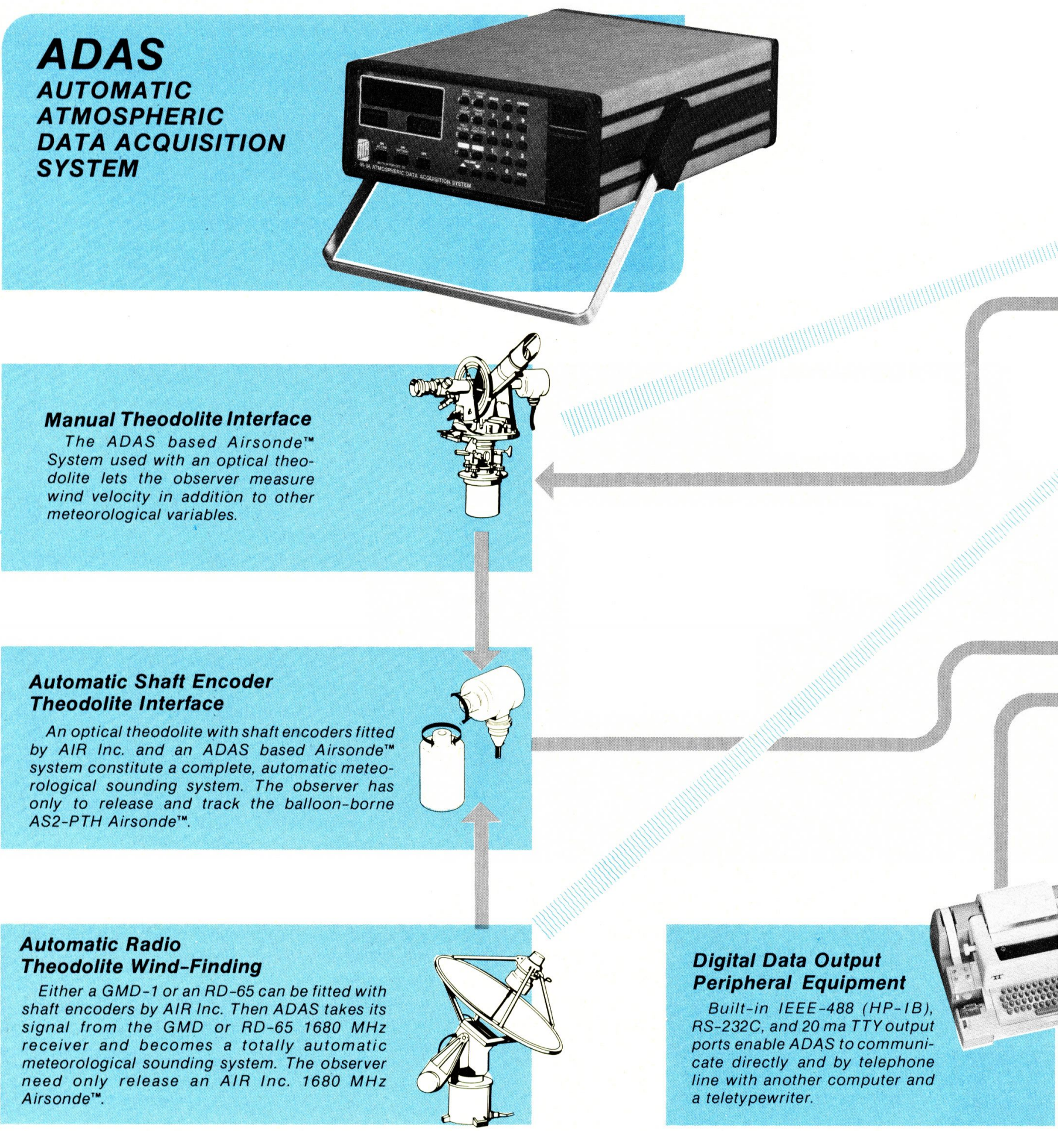

\section{Principle of Operation}

ADAS consists of a Z-80 microprocessor, several ROM chips, a programmable frequency counter, a paper tape reader, an LED display, several $1 / 0$ ports, and a theodolite interface. Each sensor used with ADAS produces a frequency that is a function of the value of the variable being sensed. ADAS measures each incoming frequency and uses calibration data entered via paper tape to calculate the value of the variable. Measured data are available in digital form in any frequently used set of units, e.g., S/ or English. The microcomputer at ADAS is programmed to calculate virtually any meteorological variable that is derivable from the standard measured variables. 


\section{Airsonde ${ }^{\mathrm{TM}}$ System}

ADAS, combined with a 403 $\mathrm{MHz}$ receiver and used with the AS2-PTH Airsonde ${ }^{\text {TM }}$, is an automatic meteorological sounding system capable of measuring vertical profiles of pressure and wet and dry-bulb temperatures and of calculating and displaying any meteorological variables derivable from the measured variables.

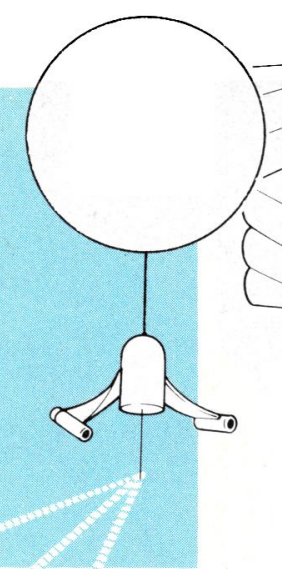

Tethersonde ${ }^{\top M}$ System

ADAS, with a $403 \mathrm{MHz}$ receiver, serves as the automatic ground station for the AIR Inc. TS-3A Tethersonde ${ }^{\text {TM }}$ System. In this system a sensor package that measures pressure, temperature, wet-bulb temperature, wind direction, and wind speed is carried aloft by an aerodynamically shaped, tethered balloon. The measured values and virtually any variable derivable from them can be automatically calculated and displayed by ADAS.

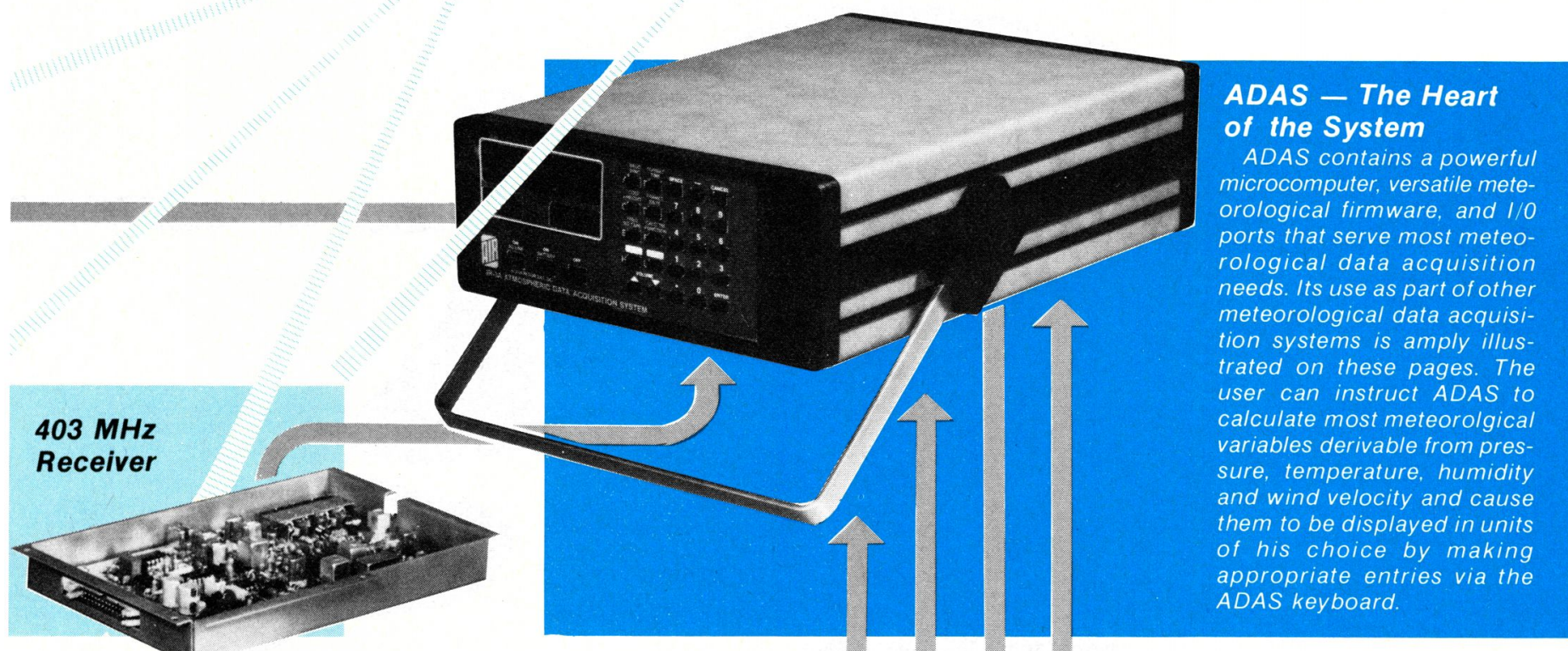

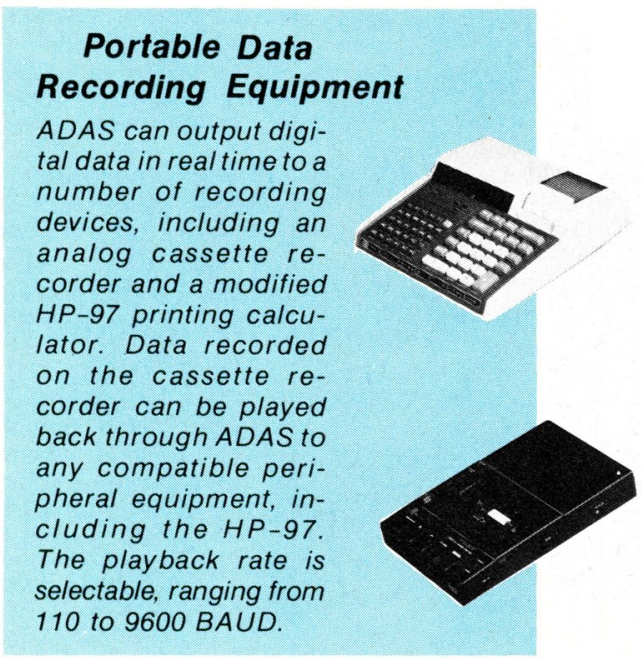

Portable Data

ADAS can output digital data in real time to a number of recording devices, including an analog cassette recorder and a modified HP-97 printing calculator. Data recorded on the cassette recorder can be played back through ADAS to any compatible peripheral equipment, including the HP-97. The playback rate is 110 to 9600 BAUD.
ADAS - The Heart of the System

ADAS contains a powerful - versatile meteports that serve most meteorological data acquisition needs. Its use as part of other meteorological data acquisisystems is amply illusthese pages. The ure, temperature, humidity appropriate entries via the ADAS keyboard.
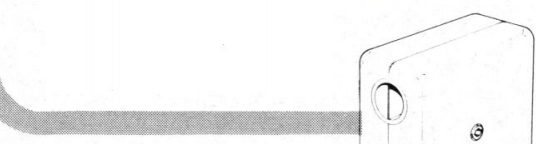
Bestsellers from

the World Meteorological

Organization available $\mathbf{O}$ from

\section{HERE IS JUST A SAMPLE \\ FROM THE RECENT BROCHURE MAILED TO AMS MEMBERS IN FEBRUARY:}

\section{Man and Climatic \\ Variability (W463)}

An informative review of current international investigations, spear headed by WMO, into climatic change and variability. Chapters discuss: global climatic change on human society; effects of human activities on global climate; the World Climate Program and relevant WMO activities. Also included is the text of the Declaration of the World Climate Conference. WMO, 1980, 32 pp., ill., $\$ 3.00$

\section{Meteorological Factors Affecting the Epidemiology of the Cotton Leaf Worm and the Pink Bollworm (W473)}

In many countries cotton is an important commercial crop, but two insects inflict considerable loss and damage. This report deals with the meteorological factors affecting the epidemiology of these two pests, the cotton leaf worm and the pink bollworm. Some recommendations are presented for improving the meteorological contributions towards understanding the epidemiology and facilitating control of both pests. The need for further research in the field and laboratory is also emphasized. WMO, 1980, 46 pp., $\$ 10.00$

\section{Applications of Remote Sensing to Hydrology (W453)}

This report deals with the application of remote sensing to the collection of data on surface waters and related waterbalance elements. The first part consists of a description of the various types of sensor, data-transmission system and remote-sensing platform which are used to obtain data on different hydrological elements. The second part of the report is devoted to the practical application of remote sensing of surface water, lakes, snow, glaciers, precipitation and soil moisture

WMO, 1979, 52 pp., ill., \$10.00
Basic Synoptic Networks of Observing Stations.

\section{Edition (W450)}

This is the latest series of network reports for each region of the World Meteorological Organization, covering the status of implementation of the Basic Synoptic Networks across the globe. The data are presented with a general introduction that provides background information on the implementa tion of the network, as well as detailed tables on operations. A full list of observational programs within the network is given along with an analysis of the pro grams' deficiencies and future plans. A set of network maps shows all stations and provides surface and upper-air charts

WMO, 1979, approx. 500 pp., $\$ 45.00$

\section{Numerical Methods Used in} Atmospheric Models, Volume 2 (W446)

A detailed account of the latest ad vances in efficient, accurate and consistent numerical methods applicable to weather forecasting. Chapters cover vertical coordinates and related discre tization; difference approximation for fluid flow on a sphere; spectral, pseudospectral, finite element and semi-implicit methods; difference approximations to quasi-geostrophic models; computational problems related to limited-area modeling; on phase speed errors due to various time differ encing schemes; and nonlinear instability.

WMO, 1979, 499 pp., $\$ 40.00$

\section{Also available}

Numerical Methods Used in Atmospheric Models, Volume 1 (W304)

WMO, 1976, 64 pp., $\$ 15.00$

\section{The Planetary Boundary}

\section{Layer (W460)}

Most human activity takes place within that part of the atmosphere next to the earth's surface which we call the atmospheric boundary layer. This re port provides a contemporary review of the current theoretical knowledge and applications of boundary layer meteor ology. Chapters cover: Ekman layer theories; tropical boundary layer, numerical modeling; operational meth ods and problems; applications of aeronautical meteorology; urban boundary layer. It will be of interest to anyone concerned with mesoscale studies evaporation, urban climatology, agrometeorology and air pollution dispersion

WMO, 1979, 201 pp., ill., \$20.00

\section{Proceedings of the World} Climate Conference: $\mathbf{A}$ Conference of Experts on Climate and Mankind (W440)

An extensive state of the art review of climate and its impact on society by a panel of leading international experts. In-depth coverage is provided for the following topics: climate and public policy; the global system that determines climate; influences of mankind on the climate system; and impacts of climate on mankind: water resources, human health, agriculture, land use, forestry, fisheries, offshore development, and world economy. WMO, 1979, 791 pp., $\$ 40.00$

Quantitative Meteorological Data from Satellites (W445)

This Technical Note provides an up to-date discussion of quantitative data from satellite measurements, including remote vertical soundings of atmospheric temperature and moisture winds derived from cloud motions observed by geo-stationary satellites; and earth surface temperature.

WMO, 1979, 102 pp., ill., \$20.00

If you haven't received your copy of our brochure, write to us.

\section{UNIPUB}

345 Park Avenue South

New York, NY 10010 